\title{
Optimal ECO-Design of Permanent Magnet Brushless DC Motor Using Modified Tabu Search Optimizer and Finite Element Analysis
}

\author{
Mohammad Yazdani-Asrami ${ }^{1 *}$, Mohammad Alipour ${ }^{2}$, and S. Asghar Gholamian² \\ ${ }^{I}$ Young Researchers and Elite Club, Sari Branch, Islamic Azad University, Sari, Iran \\ ${ }^{2}$ Faculty of Electrical and Computer Engineering, Babol University of Technology, Babol, Iran
}

(Received 2 February 2014, Received in final form 11 March 2014, Accepted 11 March 2014)

\begin{abstract}
The Permanent Magnet Brushless DC (PMBLDC) motors have been used in modern industrial factories, hazardous chemical process, modern medical devices, and especially in advanced military devices such as army drones. By considering their sensitive role in the industrial and military applications, their optimal design has a real concern. This paper proposes a method for optimal eco-design of a PMBLDC motor using improved tabu search optimization. The objective function is based on losses, volume and cost. Electrical and mechanical requirements and other limitations are combined into constraints of problem. Also, finite element analysis has been used for verifications in magnetic mode.
\end{abstract}

Keywords : BLDC, finite element method, modified tabu search, permanent magnet

\section{Introduction}

A PMBLDC motor uses permanent magnets on rotor for field excitation and electronically commutated winding on stator using hall sensors. The recent advancement in rare earth magnets helped to make PMBLDC motors efficient and compact compared to induction motor and mechanically commutated DC motors. The PMBLDC motor has high torque, compact size, and high efficiency. Therefore, this kind of motor is widely used in computers, household and industrial products, and automobiles [1-7]. Therefore, optimal design of BLDC motor has a great concern in recent years and many researchers focused on this subject. In [8], a hybrid genetic algorithm (GA) has been introduced for BLDC optimization to reduce cogging torque as electromagnetic topology optimization. A two-dimensional encoding technique, which considers the geometrical topology and a two-dimensional geographic crossover which uses as the crossover operator have introduced. A novel local optimization algorithm, called the on/off sensitivity method, hybridized with the two-dimensional encoded GA, improves the convergence characteristics. In [9], a GA based optimal design of a permanent

CThe Korean Magnetics Society. All rights reserved.

*Corresponding author: Tel: +981113239214

Fax: +981113239214, e-mail: m.yazdaniasrami@gmail.com magnet BLDC motor has been proposed considering efficiency as the objective function and motor weight and temperature rise as the constraints. In [1], a simplified analytical method to design a slotless BLDC motor has presented. This method consists of systems of equations with many approximations and in addition, this method only can be applied to small and two-pole motors. In [2], an analytical method to design and analysis of a highspeed and high-power density BLDC motor for centrifugal compressor has been introduced. Then, the electromagnetic field, back EMF, and power losses are analyzed and the results are validated by finite element method.

In most of the reviewed papers, the effect of required speed in the optimization procedure has been neglected and therefore, power of the PMBLDC motor is not well defined. In this paper, a method for the optimal design of a slotless PMBLDC motor with surface mounted magnets using modified Tabu Search (TS) has been presented considering torque, maximum speed, voltage, losses and cost. An objective function has been proposed covering the power losses, material cost and volume of the motor simultaneously, besides the mechanical and electrical requirements. All of the effective parameters and constants have been considered in the optimization problem. The TS has been used to find the optimal geometries of the assumed motor. 


\section{Introduction of PMBLDC Motor Design Formulations}

The proposed objective function is formed by a set of geometrical variables such as, number of pole pairs $(p)$, cross sectional area of the winding $\left(A_{c}\right)$, pole-arc per pole-pitch ratio $(\beta)$, magnet thickness $\left(l_{m}\right)$, stator/rotor core thickness $\left(l_{y}\right)$, winding thickness $\left(l_{w}\right)$, mechanical air gap $\left(l_{g}\right)$, rotor radius $\left(r_{r}\right)$, current density $\left(J_{c u}\right)$, wire gauge and stator/rotor axial length $\left(l_{s}\right)$, which is usually represented by the machine form factor $(\lambda)$.

$$
\lambda=2\left(r_{r}+l_{g}\right) / l_{s}
$$

Specifications that depend on materials such as winding filling factor $\left(k_{f}\right)$, permanent magnet remanence $\left(B_{r}\right)$ and stator/rotor core flux density at knee point of B-H curve $\left(B_{s y}^{k n e e}\right)$ should be given. Requirements of the motor consist of the rated value of electromagnetic torque $\left(T_{e m}\right)$ and the rated rotor rotational velocity $\left(\omega_{r}\right)$.

$$
A_{w}=\pi l_{w}\left(2 r_{r}+2 l_{g}+l_{w}\right)
$$

Where, $A_{w}$ is the winding cross sectional area. Neglecting the armature reaction and the reluctance of the stator/rotor core, the magnetic flux density at the winding surface $\left(B_{g}\right)$ is calculated as [3-5]:

$$
\begin{aligned}
& B_{g}=\frac{B_{r} l_{m}}{\left(r_{r}+l_{g}\right) \ln \left(\frac{r_{r}+l_{g}+l_{w}}{r_{r}-l_{m}}\right)} \\
& A_{g}=l_{s} \frac{\beta \pi}{p}\left(r_{r}+l_{g}\right)
\end{aligned}
$$

Where, $A_{g}$ is the air gap area at the winding surface.

The terminal voltage and current of BLDC motor can be written as [3-5]:

$$
\begin{aligned}
& \left.V=\frac{l_{s} k_{f} k_{c} A_{w}}{A_{c}}\left[1+\pi\left(r_{r}+l_{g}+l_{w}\right) /\left(p l_{s}\right)\right] \rho J_{c u}+\frac{k_{\beta} k_{1} B_{r} l_{m} \omega_{r}}{\ln \left(\frac{r_{r}+l_{g}+l_{w}}{r_{r}-l_{m}}\right)}\right] \\
& I=A_{c} J_{c u}
\end{aligned}
$$

Where, $\rho$ is the resistivity of the wire. The input power is independent of $A_{c}$, implying that the wire gauge has no effect on power of the motor as long as the winding cross sectional area is fixed. Therefore $A_{c}$ can be used to compromise between current and voltage.

The inductance of the winding $(\mathrm{L})$ is as [3-5]:

$$
L=\frac{\pi \mu_{0} k_{f}^{2} A_{w}^{2} l_{s}}{54 A_{c}^{2} p^{2} \ln \left(\frac{r_{r}+l_{g}+l_{w}}{r_{r}-l_{m}}\right)}
$$

Where, $\mu_{0}=4 \pi \times 10^{-7} \mathrm{H} / \mathrm{m}$ is free space permeability.

Power losses of a BLDC motor can be divided into three major categories: electrical, magnetic and mechanical. The power loss due to resistance of windings is considered as the most important electrical loss, which can be represented by:

$$
P_{c u}=\rho k_{f} k_{c} k_{e t} A_{w} l_{s} J_{c u}^{2}
$$

Hysteresis and eddy current losses are the dominant magnetic losses of the core material. Assuming that the air gap magnetic flux is equal to the core magnetic flux, the stator core maximum flux density due to PM can be expressed as $[3,10]$ :

$$
B_{s y}=\frac{\pi k_{1} \beta B_{r} l_{m}}{2 p l_{y} \ln \left(\frac{r_{r}+l_{g}+l_{w}}{r_{r}-l_{m}}\right)}
$$

Therefore, following expressions are obtained for hysteresis and eddy current losses:

$$
\begin{aligned}
& P_{h}=k_{h}{ }^{\prime} \rho_{y} V_{s y} B_{s y}^{n} f \\
& P_{e}=k_{e}{ }^{\prime} \rho_{y} V_{s y} B_{s y}^{2} f^{2} \\
& f=p w_{r} / 2 \pi
\end{aligned}
$$

Where, $\mathrm{f}$ is easily related to the rotational velocity, $\rho_{y}$ is material mass density and the $V_{s y}$ is the stator core volume.

The mechanical losses are categorized as windage and bearing friction. The friction of bearings can be calculated as [5]:

$$
P_{b}=\frac{N_{b}}{2} \mu_{f} F_{b} d_{i} \omega_{r}
$$

Where, $F_{b}$ is radial load of the bearing, $d_{i}$ is inner diameter of the bearing, $\mu_{f}$ is friction coefficient of the bearing, and $N_{b}$ is the number of bearings. Although, windage losses depend on the rotor parameters, it is negligible compared with other losses for a smooth cylindrical rotor, but in this paper it is consider as $5 \%$ of total loss.

Now, considering the magnetic and mechanical losses, the modified electromagnetic torque and output torque can be written as [3-5]:

$$
\begin{aligned}
& T_{e m}=\frac{\pi k_{f} k_{c} k_{1} k_{\beta} B_{r} l_{m} l_{s} l_{w}\left(2 r_{r}+2 l_{g}+l_{w}\right) J_{c u}}{\ln \left(\frac{r_{r}+l_{g}+l_{w}}{r_{r}-l_{m}}\right)}-\frac{P_{e}+P_{h}}{w_{r}} \\
& T_{\text {out }}=T_{e m}-\left(P_{w}+P_{b}\right) / \omega_{r}
\end{aligned}
$$

Where, $T_{\text {out }}$ is output torque of BLDC motor.

The volumes of permanent magnets, winding and stator/ rotor core are depending on motor geometry, so, these can 
incorporate into motor material cost. The following expression can be written:

$$
C=C_{m}+C_{w}+C_{y}
$$

Where, $C_{m}, C_{w}$, and $C_{y}$ are permanent magnet, winding and core material costs, respectively.

The cost of magnet can be divided into two categories, raw materials price and terminating process price. Raw material cost is proportional to the needed magnet volume and also, terminating process price corresponds to the finishing process, coating and adhesion procedure and is assumed to be linearly dependent on the number of pole pairs. Therefore, magnet cost expression can be written as:

$$
C_{m}=c_{m 1} \rho_{m} V_{m}+c_{m 2} p
$$

Where, $\rho_{m}, c_{m 1}, c_{m 2}$, and $V_{m}$ are the mass density of permanent magnet, the cost per unit mass of magnet, the cost corresponding to terminating process, and the volume of the magnet, respectively.

Since the price of wire per unit mass is inversely proportional to its cross sectional area, the following equation is found based on the wire price using a curve fitting approach:

$$
C_{w}=c_{1} \frac{k_{f} \rho_{w}}{A_{c}} V_{w}+c_{2} k_{f} \rho_{w} V_{w}
$$

Where, $\rho_{w}, V_{w}, c_{1}$ and $c_{2}$ are the mass density of winding, the volumes of the winding, and constants to be found using a curve fitting technique, respectively.

The cost of stator/rotor core can be easily found by knowing the selected type and thickness of laminations for stator/rotor core material. However, due to waste of materials during the punch press process, the volume of the consumed material for core should be redefined as:

$$
\begin{aligned}
& V_{t}=\pi l_{s}\left(r_{r}+l_{g}+l_{w}+l_{y}\right)^{2} \\
& C_{y}=c_{y} \rho_{y} V_{t}
\end{aligned}
$$

Where, $V_{t}, c_{y y}$ and $\rho_{y}$ are the volume of the stator/rotor core, the cost per unit mass of core, and the mass density of stator/rotor core, respectively.

\section{Overview of Tabu Search Optimizer}

The TS is an iterative optimization algorithm that first introduced by Glover in 1986 [11, 12]. TS uses memory structure and tabu list strategy that doesn't let algorithm stock in local minima. In TS we use neighborhood strategy to select the candidate list in every iteration. After making the solution list, every solution will check in tabu list, if it wasn't tabu active it could be one of the answers then we

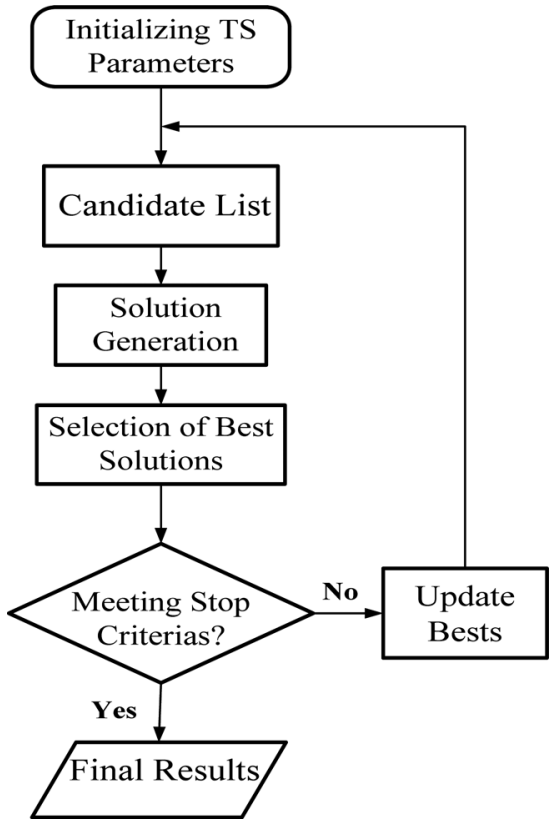

Fig. 1. The flowchart for TS algorithm.

put it in target function and if the result had proper fitness value we update the fitness condition and then we put this solution on tabu list to prevent falling in local minima or loop. Best answer in the neighborhood will be chosen as center of neighborhood in the next iteration and this will continue until stopping criteria has been satisfied. To prevent of loop or local optimum every member of tabu list will be active for constant period of iteration, after that, it will be free to select.

The flowchart of TS algorithm is shown in Fig. 1.

Application of TS in problems with many optimization parameters is so time-consuming therefore in this paper the main algorithm has been changed. So in the proposed method, the parameters have been separated in several groups to break the dimensions and decrease the solution time.

\section{The Simulation Results and Discussions}

In order to optimize the problem, first the objective function must be defined, therefore, parameters of motor have to be found, and these parameters are not constant and could be changed to optimize the problem. The parameters and their limitations represent as a vector, $\mathbf{x}$ :

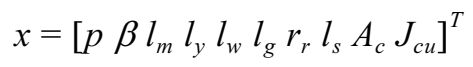

Upper and lower limits of parameters should be considered. Definition of objective function strongly depends on the field or application that this motor will be used. The important items in optimization are efficiency, volume 
and mass, robustness and performance of motor. Although in mass production, cost of materials and production are so important. To define the objective function all the parameters that effect on this important items should be considered in a way that we could get to the best result. For instance the power loss, cost and volume of motor can't be minimized all together so a trade-off between these parameters should be considered. According to the importance of these items that must be optimized a weighting factor for this items are considered and by this way these parameters are related together in objective function. So the objective function could be written as:

$$
f_{0}(x)=w_{p} \times P_{1}(x)+w_{v} \times v_{t}(x)+w_{c} \times C(x)
$$

In this equation, $w_{p}, w_{v}, w_{c}$ respectively, are weighting factor of power losses, total volume of motor and cost of used materials in the motor so, $P_{1}, V_{t}$ and $C$ are respectively power losses, total volume and cost of materials that has been used in the motor.

\subsection{Determining Optimal Value for Optimized BLDC} TS algorithm that is used to optimize this problem is a modified version of TS and is a little different with the original version. In this method the optimization parameters are separated in three groups in order to reduce the search space aspects and increase the search speed. Candidate list in this problem is consisted of optimization parameters ranges. At first the parameters will be grouped, there are ten optimization parameters and if the original version of TS would be used, it has to produce a ten aspect space, that need lots of record memory and process, so the space is broken to three groups, that firs is consisted of four aspect and others are consisted of three. Actually every candidate solution in three spaces is defined, then the first neighborhood is generated and the related answers are calculated, then all of them will be put in tabu list and become tabu active for tabu period iteration and choose the best answer to use as the neighborhood center for next iteration. In the next iteration all three aspects of answer will generate the neighborhood in its own space. Other part of process is like classic TS algorithm. To solve the optimization problem one needs to initialize the constant parameters of the optimization technique parameters which is tabulated in Tables 1 .

Table 1. Coefficient values in the TS.

\begin{tabular}{cc}
\hline \hline Control Parameters & Value \\
\hline TS period & 5 \\
TS neighborhood radius & 3 \\
TS iteration & 10 \\
\hline
\end{tabular}

To solve the optimization problem one needs to initialize the constant parameters of the motor, see Table 2. The upper and lower limits of the optimization variables and

Table 2. List of constant parameters and their values.

\begin{tabular}{cccc}
\hline \hline Parameter & Value & Parameter & Value \\
\hline$\delta$ & 5 & $\rho_{m}\left(\mathrm{~kg} \mathrm{~m}^{-3}\right)$ & 7400 \\
$B_{r}(T)$ & 1 & $\rho_{w}\left(\mathrm{~kg} \mathrm{~m}^{-3}\right)$ & 8900 \\
$B_{s y}^{\text {knee }}(T)$ & 1.5 & $\rho_{y}\left(\mathrm{~kg} \mathrm{~m}^{-3}\right)$ & 7700 \\
$\kappa\left(\mathrm{A}^{2} \mathrm{~m}^{-3}\right)$ & $10^{11}$ & $c_{m 1}\left(\mathfrak{T ~ k g}^{-1}\right)$ & 20 \\
$\rho(\Omega \mathrm{m})$ & $1.8 \times 10^{-8}$ & $c_{m 2}(\mathfrak{T})$ & 1 \\
$\left.k_{h}{ }^{\prime}(\mathrm{W} \mathrm{s} \mathrm{kg})^{-1} \mathrm{~T}^{-\mathrm{n}}\right)$ & 0.018 & $c_{y}\left(\mathrm{~T} \mathrm{~kg}^{-1}\right)$ & 3 \\
$k_{e}{ }^{\prime}\left(\mathrm{W} \mathrm{s} \mathrm{kg} \mathrm{T}^{-2}\right)$ & 0.00008 & $c_{1}\left(\mathrm{~T} \mathrm{~mm}^{2} \mathrm{~kg}^{-1}\right)$ & 0.045 \\
$n$ & 2 & $c_{2}\left(\mathfrak{T ~ k g}^{-1}\right)$ & 5.42 \\
$w_{p}$ & 0.02 & $T_{e m}^{*}(\mathrm{Nm})$ & 10 \\
$w_{v}$ & $2000 / 3$ & $w_{r}^{*}\left(\mathrm{rad} \mathrm{s}^{-1}\right)$ & 157 \\
$w_{c}$ & 0.0125 & $V^{*}(\mathrm{v})$ & 140 \\
\hline
\end{tabular}

Table 3. Optimum, upper and lower limits of the optimization variables.

\begin{tabular}{cccc}
\hline \hline Parameters & Min & Max & TS \\
\hline$P$ & 1 & 6 & 5 \\
$\beta$ & 0.5 & 1 & 0.6667 \\
$l_{m}$ & 0.001 & 0.015 & 0.103 \\
$l_{y}$ & 0.002 & 0.01 & 0.0047 \\
$l_{w}$ & 0.001 & 0.0055 & 0.0050 \\
$l_{g}$ & 0.001 & 0.004 & 0.0010 \\
$r_{r}$ & 0.005 & 0.1 & 0.0578 \\
$l_{s}$ & 0.006 & 0.6933 & 0.06850 \\
$A_{c}$ & 0.1 & 2 & 1.3677 \\
$J_{c u}$ & $3 \times 10^{6}$ & $6 \times 10^{6}$ & $5.3333 \times 10^{6}$ \\
\hline
\end{tabular}

Table 4. Characteristics of the optimized motor using TS.

\begin{tabular}{ccc}
\hline \hline No. & Parameters & TS \\
\hline 1 & $V_{t}$ & 0.0011 \\
2 & $C$ & 58.8125 \\
3 & $P_{l}$ & 61.0745 \\
4 & $P_{c u}$ & 51.8020 \\
5 & $P_{h}$ & 4.5109 \\
6 & $P_{e}$ & 2.5692 \\
7 & $P_{b}$ & 2.1195 \\
8 & $P_{w}$ & 0.0729 \\
9 & $W_{v} V_{t}$ & 0.7124 \\
10 & $W_{c} C$ & 0.7352 \\
11 & $W_{p} P_{l}$ & 1.2215 \\
12 & $W_{v} V_{t}+W_{c} C+W_{p} P_{l}$ & 2.6691 \\
13 & $f_{o}$ & 2.8536 \\
14 & Efficiency & 95.16 \\
15 & Simulation Time & 2 minutes \\
& (Convergence Speed) & \\
\hline
\end{tabular}


their obtained optimum values after optimization are listed in Table 3. Other characteristics of the motor are listed in Table 4.

The superior performance of the TS is due to its ability to simultaneously refine a local search, while still searching globally.

The proposed TS have a straight and simple structure and also low computational burden and from this point of view application of TS are very reasonable in comparison with GA, ICA, and etc. In addition based on obtained simulation results, the TS converged to the optimum solution in low iterations. Also, one of the obvious and distinguished characteristics of this algorithm is good and low standard derivation.

\subsection{Magnetic Analysis of Optimized BLDC Using 2-D FEM}

This paper uses Ansoft-MAXWELL v.14 software, which is Finite Element Method (FEM)-based software, to run the simulation for designed BLDC motor. The magnetic field inside the motor is directed by the following nonlinear partial differential equations $[13,14]$ :

$$
\begin{aligned}
& \nabla \times A=B \\
& \nabla \times(v \nabla \times A)=J \\
& \nabla \cdot \sigma\left(\frac{\partial A}{\partial t}+\nabla V\right)=0
\end{aligned}
$$

Where, $A$ is the magnetic potential vector, $J$ is the total current density, $v$ is the magnetic reluctivity, $V$ is the electric scalar potential and $\sigma$ is the electric conductivity.

In order to model BLDC motor, magnetic core and windings are primarily simulated in 2-D form, according to structural formation of the studied BLDC. For this purpose, the number of core's lamination, magnetization curve of magnetic core, turns number of windings, internal and even the material used in each element should be modeled exactly. The materials of winding and core

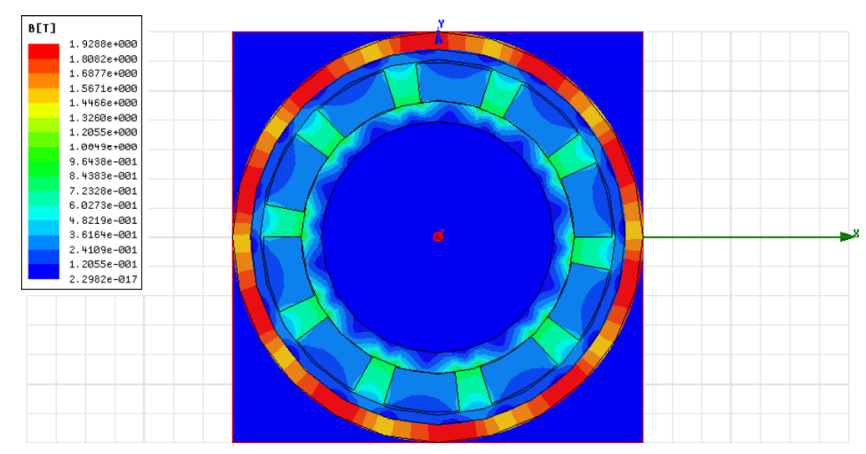

Fig. 2. (Color online) Magnetic Flux density of studied BLDC motor. laminations have been considered as Copper and M19, respectively. Also, the space between windings and core has been filled with air. It should be noted that, residual flux should be considered accurately, because of its critical impact on the magnetic field density and distribution of flux lines.

After simulation steps, the magnetic flux density of core has been shown in Fig. 2.

\section{Conclusions}

In this paper a new technique for the design optimization of PMBLDC motors has been presented considering torque, maximum speed, voltage, losses and cost. An objective function has been proposed covering the power losses, material cost and volume of the motor besides the mechanical and electrical requirements. This method is based on capability of an optimization algorithm in finding the optimal solution. For this purpose, improved tabu search optimizer has been used. The results have been analyzed and showed the efficiency of the proposed technique for design of electrical machineries.

\section{References}

[1] M. Markovic and Y. Perriard, IEEE Trans. Magn. 42, 3842 (2006).

[2] S. M. Jang, H. W. Cho, and S. K. Choi, IEEE Trans. Magn. 43, 2573 (2007).

[3] A. Rahideh, T. Korakianitis, P. Ruiz, T. Keeble, and M. T. Rothman, J. Magn. Magn. Mater. 322, 3680 (2010).

[4] J. M. Seo, J. H. Kim, I. S. Jung, and H. K. Jung, IEEE Trans. Ind. Appl. 47, 730 (2011).

[5] S. H. Lai, Ph.D. Thesis, University of Southampton, England (2006).

[6] Y. U. Park, J. H. Cho, S. H. Rhyu, and D. K. Kim, J. Magn. 18, 117 (2013).

[7] J. W. Jung and T. H. Kim, J. Magn. 15, 40 (2010).

[8] C. H. Im, H. K. Jung, and Y. J. Kim, IEEE Trans. Magn. 39, 2163 (2003).

[9] P. R. Upadhyay, and K. R. Rajagopal, J. Appl. Phys. 97, 516 (2005).

[10] D. Lee, Y. J. Kim, and S. Y. Jung, J. Magn. 18, 216 (2013).

[11] F. Glover, and M. Laguna, Tabu Search, University of Boulder (1997).

[12] A. Hertz, E. Taillard, and D. De Werra, A Tutorial on Tabu Search, OR Spektrum (1989) pp. 131-141.

[13] J. P. A. Bastos and N. Sadowski, Electromagnetic Modeling by Finite Element Methods, Marcel Dekker Inc. (2003).

[14] R. Bargallo, Finite Elements for Electrical Engineering, Universitat Politecnica De Catalunya (2006). 